\title{
Analgesia obstétrica: ¿Una práctica deseable?
}

\author{
Patricia Vélez Jaramillo*
}

\section{RESUMEN}

El propósito de la revisión es describir los mecanismos y efectos relacionados con el dolor durante el trabajo de parto, así como evaluar las ventajas y efectos secundarios de la analgesia peridural, como una de las primeras alternativas en el manejo del dolor en obstetricia.

PALABRAS CLAVES: Analgesia para el parto: técnica peridural, opioides: fentanyl, sufentanil, anestésicos locales: bupivacaina.

\section{SUMMARY}

The purpose of this review is describe the mechanisms and related effects with the pain in labor so how evaluate advantajes and side effects of peridural analgesia how one of the first selection technics in the management of pain in labor.

KEY WORDS: labor analgesia: epiduraI techniques, opioids: fentanyl, sufentanil, local anesthetic: bupivacaina.

El dolor ha sido definido como una compleja y subjetiva constelación de experiencias sensoriales, perceptuales y emocionales, asociada con variadas respuestas autonómicas, sicológicas y de comportamiento que se desencadenan como respuesta al estímulo nociceptivo generado por la lesión tisular.

El dolor asociado con el parto afecta a todas las pacientes en diversos grados y su complejidad involucra alteraciones bioquímicas y fisiológicas que afectan no solamente a la madre sino también al feto, e interactúan interfiriendo con la evolución normal del trabajo de parto.

La injuria tisular desencadenada por la isquemia de la contractilidad uterina, como uno de los mecanismos fisiopatológicos implicados, lleva a la sensibilización de los nociceptores tisulares periféricos y sus fibras nerviosas aferentes, con la consecuente liberación de neurotransmisores, excitatorios e inhibitorios como sustancia $\mathrm{P}$, neurotensina, encefalinas, Gaba, prostaglandinas y otras. Las vías del dolor efectúan su primera sinapsis en interneuronas del cuerno posterior de la medula espinal, donde se realiza la neuromodulación del estímulo nociceptivo e interactúan con otras neuronas en el asta anterior medular y en segmentos localizados en zonas adyacentes, activando vías ascendentes a nivel del tallo y la corteza, desencadenando múltiples respuestas reflejas, de tipo sicológico, sensorial, cognitivo, afectivo y autonómico. La estimulación autonómica especialmente de tipo simpático, aumenta la actividad respiratoria y

Médica anestesióloga. Profesora Asistente. Departamento Clínico Quirúrgico. Facultad de Ciencias para la Salud. Universidad de Caldas. circulatoria y origina mecanismos sicodinámicos como aprehensión y ansiedad.

Se genera alcalosis respiratoria y posteriormente acidosis

metabólica por el incremento en la ventilación durante la contracción y los periodos de hipoventilación e hipoxemia durante la relajación uterina. Aumentan la presión sistólica y la frecuencia cardíaca, así como el gasto cardíaco, el trabajo ventricular izquierdo, y el consumo de oxígeno. La actividad metabólica aumentada se manifiesta por niveles elevados de lactato y ácidos grasos libres. La motilidad intestinal, y el vaciamiento gástrico se disminuyen y los niveles de gastrina se incrementan.

Como mecanismos desencadenantes de esta respuesta están: las catecolaminas, el cortisol, los corticosteroides y la ACTH, liberados como manifestación neuroendocrina. Estos cambios pueden alterar el flujo sanguíneo uteroplacentario, lo que incide sobre el intercambio gaseoso, la oxigenación fetal y del recién nacido. En muchos casos los cambios generados por el dolor pueden originar un trabajo de parto disfuncional con prolongación del parto.

Posterior al parto, la experiencia dolorosa puede desencadenar alteraciones emocionales, como depresión, ansiedad y disfunción sexual (1).

El dolor durante el trabajo de parto tiene un componente somático y visceral, relacionado con la activación de nociceptores y reflejos espinales desencadenados en órganos como: el útero, el cérvix, los músculos abdominales, el periné y las estructuras osteoarticulares de la pelvis. La nocicepción es variable, dependiendo de múltiples factores cómo: edad, paridad, raza, nivel socio-económico, estadio del parto y complicaciones del mismo entre otros. El estímulo doloroso es transmitido a los segmentos epinales torácicos $\mathrm{T} 10$ - 12 y lumbares L1, en su primera fase, e involucra 
además las fibras sacras 2 - 4 en su segunda fase. Las características de estas ultimas fibras nerviosas mas gruesas, así como la sinapsis a nivel medular explica por que estas, son mas difíciles de bloquear persistiendo la sensación de presión a nivel del canal del parto en el momento del expulsivo (1-2).

Actualmente se dispone de varias alternativas analgésicas para el manejo del dolor durante el trabajo de parto; técnicas intravenosas con narcóticos, técnicas inhalatorias, bloqueos regionales y técnicas peridurales y espinales. Sin embargo solo estas ultimas han demostrado controlar en forma efectiva el dolor, hecho respaldado por el alto grado de satisfacción de las pacientes y las mediciones de variables hemodinámicas y respiratorias; observándose además condiciones de seguridad para la madre, el feto y el recién nacido.

En esta revisión nos referiremos sólo a las técnicas peridurales simples o combinadas

Técnicas

I- Analgesia peridural:

1. Dosis única

2. Bolos intermitentes

3. Infusión continua

4. Analgesia peridural controlada por el paciente (PCA).

II- Analgesia combinada peridural y espinal

III- Analgesia espinal de dosis única

I- Analgesia peridural: es la administración de una mezcla de anestésico local y / o narcótico en el espacio epidural, la cual puede ser administrada como dosis única (no muy recomendada por su limitación analgésica), o en bolos intermitentes a través de un catéter ubicado en el mismo espacio. Las dosis pueden ser también administradas en forma continua mediante bomba de infusión. Las dosis bolo serán aplicadas periódicamente según el nivel de analgesia de la paciente. Sin embargo la concentración sanguínea en picos es una de sus desventajas.

En la analgesia peridural continua se administra una dosis de carga y se inicia una infusión constante calculada previamente. Permite mantener una concentración sanguínea constante y la analgesia perineal es mas significativa. El bloqueo del estimulo nociceptivo se logra por el efecto a nivel de las raíces nerviosas y de los receptores del asta dorsal de la medula espinal. El período de latencia para una efectiva analgesia puede tardar 20 minutos.

PCA: Esta técnica es similar a la PCA IV. La madre se auto administra a través del catéter peridural pequeños bolos de la mezcla de anestésico local mas opioide, en una cantidad y dosis limitada, la cual se ha programado previamente en una bomba de infusión que administra además un goteo continuo. La autonomía de la paciente en la entrega de la droga, hace que esta sea titulada, con buena calidad analgésica, pocos efectos colaterales, dosis total final baja y buena satisfacción personal $(2-6,10,28)$.

\section{II - Analgesia combinada peridural y raquídea:}

como su nombre lo indica, esta combina las ventajas de ambas técnicas. Se logra mediante la administración subaracnoidea de una dosis baja de un opioide, que tiene la capacidad de producir una analgesia profunda, de inicio casi inmediato y duración intermedia (dependiendo del medicamento empleado, 2.5 - 3 horas si se emplea el fentanylo sufentanyl). Simultáneamente se dejainstaurado un catéter a nivel peridural para la administración de las dosis posteriores, en bolos o en infusión continua de bupivacaina sola o asociada a opioide.

Estas dosis subaracnoideas pequeñas de medicamentos permiten preservar la actividad motora y la deambulación de las pacientes, con un alto grado de satisfacción de ellas. Actualmente es la técnica que muestra mayores ventajas y es altamente recomendada por la flexibilidad y el mantenimiento de la analgesia por periodos prolongados. Efectos secundarios como hipotensión, depresión respiratoria, náusea y vómito son muy raros $(2-6,10-31)$.

III - Analgesia raquídea de dosis única: es la administración de una dosis única en el espacio subaracnoideo, de anestésico local mas narcótico. Se utiliza generalmente en estadios avanzados de la dilatación. . No es muy recomendada, en razón a que no permite administrar nuevas dosis, a menos que se repita la punción $(2-6,10)$.

\section{Interrogantes}

Al elegir una modalidad analgésica deben considerarse aspectos cómo: estado clínico de la paciente, patología asociada, características del trabajo de parto, dilatación cervical, parto inducido o espontáneo, estado fetal, duración de la analgesia, latencia de la técnica y la disponibilidad tecnológica (bomba de infusión, agujas, catéteres y medicamentos).

Muchos obstetras son renuentes a emplear estas técnicas, por diversas razones entre las que pueden mencionarse: desconocimiento de ellas, ideas erróneas con respecto a sus posibles efectos sobre el trabajo de parto y el expulsivo y presiones laborales que impiden una mayor disponibilidad de tiempo para supervisar la paciente con analgesia.

Un gran número de investigaciones han pretendido resolver los diversos interrogantes planteados por obstetras y anestesiólogos con respecto a la analgesia y su asociación con una mayor frecuencia de parto instrumentado, la prolongación del trabajo de parto, los mayores requerimientos de oxitocina para reforzar el trabajo de parto, la mayor incidencia de cesárea y los posibles efectos sobre el recién nacido $(2,5-6)$.

Aunque existen investigaciones que demuestran que la analgesia peridural podría tener efectos adversos en relación a estos últimos interrogantes, muchas otras debaten y contradicen estas observaciones, basadas en estudios multicéntricos, controlados, randomizados, prospectivos, doble ciegos y de alta significancia estadística. Desventajas atribuidas a la analgesia peridural, como prolongación del primero y segundo estadio del trabajo de parto y la frecuencia mayor de parto instrumentado han sido explicadas por las dosis altas de medicamentos que anteriormente se utilizaban y que hoy han sido reemplazadas por concentraciones mas diluidas de anestésicos locales, así como por la administración de dosis menores de narcóticos sintéticos. Se ha visto además que las pacientes con analgesia pueden tener una prolongación de la fase del expulsivo hasta de tres horas, sin que ello represente mayor morbilidad. 
Aunque inicialmente algunos estudios mostraron una incidencia mayor de cesáreas por distocia en primigestantes, cuando la analgesia se había administrado en una dilatación menor de $5 \mathrm{~cm}$, nuevos datos contradicen esta idea. Chesnut realizo dos trabajos en los que no se demuestran diferencias en relación a la incidencia de cesáreas, en nuliparas con parto inducido o espontáneo, cuando la analgesia se había iniciado en una dilatación menor de $4 \mathrm{~cm}$. Existen además reportes de servicios de obstetricia, donde la practica rutinaria de analgesia peridural no ha variado el índice de cesáreas e incluso aun, lo ha disminuido. Con respecto al uso de la oxitocina, su utilización de acuerdo con protocolos establecidos para su uso racional no se ha asociado con efectos nocivos matemos ni fetales (14).

La evaluación del monitoreo fetal posterior a la analgesia puede mostrar una variabilidad disminuida transitoria inicial que se recupera posteriormente. Este hecho se ha correlacionado con estudio de gases arteriovenosos en sangre de cordón al nacimiento, los cuales no han mostrado evidencia clínica compatible con depresión neonatal y acidosis; igualmente no se han demostrado efectos desfavorables sobre el puntaje de apgar o los parámetros de neurocomportamiento en el período neonatal inmediato (2, 4-6, 13-16, 19-20).

En relación a los efectos colaterales que pueden presentarse con la administración de estas técnicas, se ha visto que la hipotensión es fácilmente prevenible y de rápido manejo cuando se presenta. El prurito es transitorio y auto limitado y rara vez requiere intervención farmacológica.

La depresión respiratoria es muy rara y se ha asociado a sobredosificación o al empleo simultáneo de narcóticos parenterales (22).

La analgesia peridural es un procedimiento médico que ha sido bien documentado $\mathrm{y}$ ha probado ser muy seguro. Es verdad que puede tener algunos efectos y complicaciones pero son previsibles y excepcionalmente serios $(2,4-9,11,13$, 19).

\section{Beneficios}

Los opioides tienen un comportamiento díferente a los anestésicos locales, ya que no interrumpen la transmisión nerviosa; no causan bloqueo motor, ni interfieren con otros tipos de sensación excepto el dolor. Cuando se administran solos son insuficientes para contrarrestar el dolor totalmente, por lo que se recomienda emplearlos con anestésicos locales, con lo que se logra disminuir las dosis requeridas, acortar la latencia, aumentar la duración, mejorar la calidad de la analgesia y disminuir así la incidencia de efectos secundarios, lográndose además un paso transplacentario menor al feto. $(2,18)$. Ventajas documentadas indican un alivio efectivo del dolor, sin causar sedación o confusión mental. La posibilidad de extender la analgesia en caso de cesárea o parto instrumentado, así como la posibilidad de continuar la analgesia postoperatoria, son condiciones que hacen más deseable su utilización. Existen además complicaciones médicas asociadas al embarazo que justifican aún más la utilización de estas alternativas de analgesia, como son los casos de hipertensión arterial, parto prematuro, embarazo gemelar, parto en pelvis, parto vaginal posterior a una cesárea previa y muchas otras (2).

El bloqueo de la respuesta neuroendocrina al estress, el control de los parámetros respiratorios, de las variables hemodinámicas, la mejoría de la oxigenación y del flujo intervelloso, están suficientemente respaldadas por mediciones clínicas y de laboratorio. $(2-3,13)$.

\section{Problemas asociados}

Cefalea: la incidencia de cefalea es muy baja, aunque se asocia mas frecuentemente a las técnicas combinadas, la mayoría de las veces de poca intensidad y con buena respuesta a mediadas sencillas cómo analgésicos y reposo $(2,5-6,11)$.

Dolor lumbar: es muy común después del embarazo y el parto. La molestia asociada a la punción usualmente no dura mas de una semana.

Lesión de los nervios: la lesión secundaria a las técnicas peridurales pueden ocurrir, pero son muy raras y su recuperación se logra espontáneamente la mayoría de las veces. Se mencionan complicaciones asociadas al parto como resultado de la tracción o presión directa de la cabeza fetal o a la lesión directa por fórceps.

El riesgo de lesión neurológica residual es remoto; se ha relacionado con la presencia de transtomos de la coagulación, pacientes anticoagulados, infección, y a la existencia de preservativos o contaminantes en las soluciones $(2,5-6,11)$.

Hipotensión: recomendaciones como el aporte previo de cristaloides, el empleo de vasopresores, y la desviación del útero a la izquierda, hacen de esta una manifestación de rápido y fácil manejo.

Otros efectos como: prurito, escalofrío, distensión de la vejiga y debilidad en miembros inferiores pueden ser efectos transitorios y de poca importancia ( $2,5-6,11)$.

Efectos fetales: Las catecolaminas liberadas durante el trabajo de parto como resultado del dolor y la ansiedad tienen un efecto negativo para la madre y el feto. La hiperventilación puede conducir a alcalosis y por tanto a una aporte menor de oxígeno para el feto por desviación de la curva de la oxihemoglobina. Se ha demostrado que los narcóticos empleados en condiciones normales $y$ en las dosis recomendadas, no tienen efectos a nivel de la circulación fetal durante el trabajo de parto. Es conocido que la mayoría de medicamentos tienen de acuerdo a sus propiedades farmacológicas, la capacidad de pasar al feto, sin que ello implique un efecto nocivo. Sustancias comunes como la cafeína $y$ el alcohol ingeridos previamente al parto, pueden ser detectadas en el recién nacido durante horas. El monitoreo electrónico de la frecuencia cardíaca fetal puede mostrar una disminución transitoria de la variabilidad, sin que ello se asocie con alteraciones patológicas de feto o recién nacido $(2,5-6,11$, 13).

\section{Discusión}

Múltiples razones inciden en la interpretación de los datos de las diversas investigaciones debido al tipo de población estudiada, institucional o particular, dinámica de los grupos de trabajo, tipo de estudios realizados, 
homogeneización de las características de la muestra y muchas otras.

La revisión bibliográfica muestra una gran diversidad de estudios donde se comparan medicamentos, dosis y técnicas peridurales y raquídeas. La mayoría respaldan la seguridad y coinciden en los grandes beneficios de estas técnicas, sin disponer hasta la fecha de alternativas comparables.

La analgesia peridural puede asociarse en algunos casos con prolongación del segundo período del parto; sin embargo esto no parece asociarse a complicaciones maternas ni fetales. El estado fetal satisfactorio evaluado a través del monitoreo de la frecuencia cardíaca fetal y del perfil biofísico fetal y su relación posterior con mediciones de gases arterio-venosos en sangre del cordón, respaldan este hecho al no mostrar evidencia clínica compatible con acidosis. La evaluación del monitoreo fetal posterior a la analgesia puede mostrar una variabilidad disminuida transitoria inicial que se recupera posteriormente. Igualmente no se han demostrado efectos desfavorables sobre el puntaje de apgar o los parámetros de neurocomportamiento en el período neonatal inmediato. Aunque estas técnicas de analgesia no se han relacionado con alteraciones del neurocomportamiento, se requieren estudios de seguimiento de los recién nacidos por períodos más prolongados (2, 5-6).

Chestnut en algunos estudios demuestra que los requerimientos de oxitocina y la incidencia de malposición del vertex, no se incrementan en pacientes con analgesia temprana, cuando se administra en la fase activa y durante el trabajo de parto espontáneo. Sin embargo algunos centros recomiendan como política general administrar la analgesia en primigestantes en fase activa $y$ en una dilatación cervical mayor de $5 \mathrm{~cm}$. Se requieren estudios posteriores para evaluar el efecto de la analgesia en pacientes con trabajo de parto inducido (14-15).

En conclusión, las técnicas de analgesia peridural son efectivas en el alivio del dolor durante el trabajo de parto, en el sentido de que no se pretende abolir totalmente la sensación dolorosa. La calidad y efectividad de la analgesia dependen no solamente de los medicamentos empleados, dosis, técnica, fase del trabajo de parto y dilatación sino también de las condiciones sicoafectivas y umbral al dolor.

La administración de una infusión continua peridural en baja concentración, acompañada de la administración inicial de narcótico espinal, parece ser la técnica de elección, en razón a la calidad analgésica alcanzada y la menor interferencia con el trabajo de parto. Definitivamente la adición de opioides permite bloquear en forma selectiva el estimulo doloroso sin interferir con la actividad motora; disminuye además las dosis de anestésicos locales requeridos, acorta la latencia, mejora la calidad y prolonga la analgesia, produce menor bloqueo motor, las dosis totales requeridas son mas bajas y los efectos secundarios a nivel materno y fetal son menores. Los múltiples efectos benéficos para la madre y el feto, que pueden ser evaluados mediante variables hemodinámicas, respiratorias y parámetros de oxigenación, respaldan este hecho; demostrándose además una satisfacción materna significativa.

El fentanyl intratecal en dosis de 25 microgramos, o sufentanil 10 microgramos, asociado a pequeñas dosis peridurales de bupivacaina en concentraciones de 0,0625 $0,25 \%$, ha demostrado ser en la actualidad una de las alternativas de elección en el control del dolor durante el trabajo de parto (12, 17-18, 32).

Como todo procedimiento médico existen riesgos y en algunos casos pueden presentarse efectos secundarios; aunque la mayoría de ellos son de fácil manejo. Sin embargo cuando los beneficios son evidentes, las técnicas están realmente justificadas. Es también conocido que el empleo de estos procedimientos requiere un personal especializado y que la aplicación cada vez más frecuente de estos permitirá un están dar alto de entrenamiento y el fortalecimiento de los servicios de analgesia obstétrica.

Se han reportado unos pocos casos de depresión respiratoria materna con el uso de sufentanil intratecal; dicha complicación se ha asociado al empleo simultáneo de narcóticos por vía parenteral.

Como una desventaja se menciona, el bloqueo insuficiente a nivel del recto y vagina, manifestado como "sensación de presión", pero la conservación del reflejo de pujo debe verse más como algo ventajoso pues facilita el expulsivo. Se recomienda además el empleo suplementario de anestésicos locales a nivel de la episiorrafía.

Pese al análisis imparcial de los estudios y a la validez de los mismos, existen polémicas por parte de obstetras y anestesiólogos, la mayoría de las veces motivadas en desconocimiento o desacuerdo injustificado con las técnicas. No parece quedar duda sin embargo de la gran utilidad de la analgesia obstétrica y de los grandes beneficios que ofrece a la madre.

\section{BIBLIOGRAFIA}

1. Bonica J, McDonald J. The pain of childbirth. En: Bonica J, ed. The management of pain second ed. Philadelphia Lea Febiger 1990; (2): 131343.

2. Brownridge P, Mears N, Watterson L, Ward E. Pain relief and anesthesia in childbirth. 1999 Junio 20. A vailable from: URL: http:/ /www.mambit.com/obstestspain/oapil.htm

3. Halpern S, Leighton B, Ohlsson A y col. Effect of epidural vs parenteral opioid analgesia on the progress of labor. Jama. 1998; (280): 2105-10.

4. Gambling D. Labor analgesia: advances and autcomes. 1998 jul 19. Available from: URL: www.imhotep.net/lecture4.html
5. Thorp J. Epidural analgesia in labor. Clark A. Epidural analgesia: a subject of controversia. Pro/Con clinical forum. Labor and epidural a analgesia. Available from: URL: http://www.soap.org/ archives/epilabor htm.

6. Vincent R, Chestnut D. Epidural analgesia during labor. 1999 Nov 15 Available from: URL: http://www.aafp.org/afp/981115 ap/vincent.html.

7. Cerda S. ¿Qué hay de nuevo en anestesia regional obstétrica? Rev Col Anets 1998; 26: 25-32.

8. Cerda S. Analgesia en trabajo de parto: epidural o combinada espinalperidural ? Rev Col Anets 1998; 26: 45-56. 
9. Tan S, Reid J, Thorburn J. Extradural analgesia in labor: complications of three tecniques of administracion. Br. J. Anaesth 1994; 73: 619-23.

10. Hugens S. Analgesia methods during labour and delivery. Can J Anaesth 1992; 39 (5): 18-23.

11. Chaney M. Side effects of intrathecal and epidural opioids. Can J Anaesth 1995; 42 (10): 891-03.

12. Palmer C, Cork R, Hays R, y col. The dose response relation of intrathecal fentanyl for labor analgesia. Anesthesiology 1998; 88: 355-61.

13. Palmer C, Maciulla J, Cork R, Y col. The incidence of fetal heart tale changes after intrathecal fentanyl labor analgesia. Anesth Analg 1999; 88: 577-81.

14. Shennan A, Smith R, Browne D Y col. The elective use of oxytocin infusion during labour in nulliparous women using epidural analgesia. Int $\mathbf{J}$ Obstet Anesth 1993; 2: 15-20.

15. Chestnut D, McGrath J, Vincent R y col. Does early administration of epidural analgesia affect obstetric outcome in nulliparous women who are in spontaneous labor? Anesthesiology 1994; 80: 1201-08.

16. Dewan D, Caben S. Epidural analgesia and the incidence of cesarean section: time for a closer lock. Anesthesiology 1994; 80: 1189-92.

17. Herman N, Calicott R, Van Decar $\mathrm{T} y$ col. Determination of the dose response relationship for intrathecal sufentanil in laboring patients. Anesth Analg 1997; 84: 1256-61.

18. Caben S, Amar D, Pantuck C y col. Epidural analgesia for labor and delivery: fentanyl o sufentanil? Can J Anaesth 1996; 43 (4): 341-6.

19. Macdonald R. Problems with regional anaesthesia: hazard or negligence? Br. J. Anaesth 1994; 73: 64-68.

20. Viscomi C, Rathmell J, Pace N. Duration of intrathecal labor analgesia: early versus advanced labor. Anesth Analg 1997; 84: 1108-12.
21. D' Angelo R, Anderson Mphillip J, y col. Intratecal sufentanyl compared to epidural Bupivacaina for labor analgesia. Anesthesiology 1994; 80: 120915.

22. Katsiris S, Williarns S, Leighton B, Halpern S. Respiratory arrest following intrathecal inyection of sufentanil and bupivacaina in a parturient. Can J Anaesth 1998; 45(9): 880-83.

23. Lions G, Columb M, Hawthorne L, Dresner L. Extradural pain relief in labour: bupivacaina sparing by extradural fentanyl is dose dependent. Br J Anaesth 1997; 78: 493-97.

24. Douglas M. Alternatives to epidural analgesia during labor. Can J Anaesth 1991; 38(4): 421-4.

25. Oyston J. Obstetrical anaesthesia in Ontario. Can J Anaesth 1995; 42 (12): 1117-25.

26. Groves E, Groves J. Epidural analgesia for labour in a patient with Ebsteins anornaly. Can J Anaesth 1995; 42(1): 77-9.

27. Pattee $\mathrm{C}$, Penning. Obstetrical analgesia in a parturient with antithronibin III deficiency. Can J Anaesth 1993; 40(6): 507-10.

28. Kleiman S, Wiesel S, Tessler M. Patient controlled analgesia (PCA) using fentariyl in a parturient with a platelet function abnormality. Can $\mathbf{J}$ Anaesth 1991; 38(4): 489-91.

29. Rosaeg O, Kitts J, Koren G. Maternal and fetal effects of intravenous patient controlled fentanyl analgesia during labour in a thrombocytopenic parturient. Can J Anaesth 1992; 39(3): 277-81.

30. Morris G, Lang S, Yip R. Can parturients distinguish between intravenous and epidural fentanyl? Can J Anaesth 1994; 41(8): 667 - 72.

31. Abouleish A, Abouleish E, Camann W. Combined spinal-epidural analgesia in advanced labor. Can J anaesth 1994; 41(7): 575-8.

32. Singh H, Yang J, Thornton K, Giesecke A. Intrathecal fentanyl prolongs sensory bupivacaina spinal block. Can J Anaesth 1995; 42(11): 987-91. 